\title{
DESENVOLVIMENTO DO PERFIL SENSORIAL E ACEITAÇÃO DE REQUEIJÃo CREMOSO' ${ }^{1}$
}

\author{
Deborah dos Santos GARRUTI ${ }^{2, *}$, Edy Sousa de BRITO², Telma M. BRANDÃO², \\ Plínio UCHÔA Jr. ${ }^{2}$, Maria Aparecida A. P. da SILVA ${ }^{3}$
}

\begin{abstract}
RESUMO
O perfil sensorial de requeijão cremoso foi desenvolvido por meio da Análise Descritiva Quantitativa (ADQ) utilizando-se quatro marcas comerciais do produto. Onze termos descritivos foram desenvolvidos por uma equipe de provadores selecionados, que geraram também a definição de cada termo e as amostras-referência. Os dados obtidos foram submetidos a ANOVA, teste de Tukey e Análise de Componentes Principais. O requeijão da marca A caracterizou-se por apresentar baixa consistência e aparência lisa, enquanto a característica predominante na marca B foi o sabor salgado muito intenso. Os produtos das marcas C e D apresentaram perfis sensoriais semelhantes entre si e distintos da marca A, por serem mais consistentes. Foi também realizado um teste de aceitação utilizando escala hedônica de nove pontos. Todas as marcas apresentaram alta aceitabilidade global, não apresentando diferença significativa entre si. A aceitação dos consumidores foi baseada no manuseio do produto, os quais gostaram mais das amostras mais consistentes e com menor formação de fio.

Palavras-chave: requeijão cremoso; controle de qualidade; análise sensorial; análise descritiva quantitativa; aceitação.
\end{abstract}

\section{SUMMARY}

SENSORY PROFILE AND ACCEPTANCE OF "REQUEIJÃO CREMOSO" CHEESE. "Requeijão cremoso" is a type of smooth and spreadable cheese, manufactured with milk coagulated by natural acidification and addition of milk cream or butter oil. The sensory profile of four commercial brands of requeijão cremoso was developed through Quantitative Descriptive Analysis (QDA). Eleven descriptive terms, their definitions and reference samples were generated by a selected and trainned panel. The statistical analysis included Analysis of Variance (ANOVA), Tukey's Test for means, and Principal Component Analysis (PCA). Brand A showed low consistency and a smooth appearance. The main attribute of brand B was a strong salty taste. Both products $\mathrm{C}$ and D showed very similar sensory profiles, presenting higher consistency than brand A. Laboratory consumer tests were also carried out, using a nine point hedonic scale. All products showed good acceptance, but subjects preferred the samples with higher consistency.

Keywords: requeijão cremoso; quality control; sensory analysis; descriptive quantitative analysis; acceptance.

\section{1 - INTRODUÇÃO}

A tecnologia dos queijos fundidos surgiu no início do século XX, como uma necessidade de se deter os processos microbianos e enzimáticos de queijos suiços e alemães, de forma a viabilizar a exportação para países de clima quente. Em 1911, Gerber e Stettler conseguiram solubilizar o paracaseinato de cálcio da matéria-prima por meio de calor, usando citrato de sódio como agente fundente, obtendo assim o que foi chamado de queijo processado. O sal sódico, sob agitação constante e calor, promove uma troca interna de ions, transformando o paracaseinato de cálcio, de hidratação instável, em paracaseinato de sódio, cuja solução é coloidal e estável [15].

Requeijão cremoso é um tipo de queijo fundido cremoso, obtido por fusão de uma massa de coalhada dessorada e lavada obtida por coagulação ácida e/ou enzimática do leite, com adição de creme de leite e/ou manteiga e/ou gordura anidra de leite e/ou butter oil [3]. Recentemente a indústria brasileira adotou o processo de acidificação direta combinado com precipitação pelo calor [8]. A composição de um requeijão cremoso típico

\footnotetext{
Recebido para publicação em 18/06/2002. Aceito para publicação em 02/04/2003 (000857).

${ }^{2}$ EMBRAPA Agroindústria Tropical, Av. Dra Sara Mesquita, 2270, CX Postal 3761,CEP 60511-110,Fortaleza,CE.E-mail: deborah@cnpat.embrapa.br ${ }^{3}$ Faculdade de Engenharia de Alimentos - FEA, UNICAMP, Depto. de Planejamento Alimentar e Nutrição - Cx Postal 6121, CEP 13083-970, Campinas, SP.

* A quem a correspondência deve ser enviada.
}

consiste em 58-60\% de água, 24-27\% de gordura, 9-11\% de proteína, $1-2 \%$ de carboidratos e $1-1,5 \%$ de $\mathrm{NaCl}$ [7].

Alguns dos defeitos mais comuns em queijos fundidos cremosos são: consistência granulada; fluidez heterogênea; fluidez excessiva; emulsão brilhosa ou com separação de gordura; descoloração ou escurecimento do produto; textura arenosa, cristalizada; defeitos de sabor, como sabor fraco, picante, amargo, rançoso, ácido, químico, alcalino e metálico. No entanto, são poucos os estudos de avaliação sensorial do requeijão brasileiro que contemplam uma análise descritiva dos parâmetros de qualidade.

FERNANDES et al. [6], estudando a possibilidade de utilização de novos sais emulsificantes para a elaboração de requeijão cremoso, utilizaram metodologia de Kramer para comparar as formulações desenvolvidas com um requeijão padrão, bem como uma escala hedônica de 7 pontos para avaliar a aceitação em termos de sabor, corpo e textura. MUNCK et al. [11] avaliaram a qualidade de requeijão obtido do leite coalhado com ácido cítrico e fundido com citrato de sódio, em termos de consistência, textura (ausência de grumos), viscosidade, untabilidade e sabor, porém não reportaram a metodologia utilizada.

GIGANTE et al. [8] avaliaram a aceitabilidade de requeijão adicionado de concentrado protéico de soja quanto ao sabor, espalhabilidade, cremosidade, firmeza, cor e aceitabilidade global, a qual foi também avaliada pelo Mapa de Preferência Interno. O produto apresentou boa aceitabilidade, atingindo valores hedônicos entre 6 a 8, em uma escala de 9 pontos. 
Estudos de estabilidade de requeijão têm utilizado a análise sensorial para determinar a vida-de-prateleira desse produto. VAN DENDER et al. [16] avaliaram aroma, cor, odor e textura de requeijão embalado em cinco diferentes embalagens. Os produtos não apresentaram diferença significativa entre si quanto aos parâmetros estudados, sendo preservados até 75 dias a $10^{\circ} \mathrm{C}$.

Com a finalidade de estabelecer a qualidade sensorial de requeijão cremoso tradicional e "light", CASTRO et al. [4] desenvolveram um Perfil de Caracteristicas [1] por meio de escala de cinco pontos para aparência, cor, odor, sabor e cremosidade. As quatro amostras avaliadas (duas tradicionais e duas "light") apresentaram diferença apenas para odor e sabor.

Em uma avaliação comparativa das características do requeijão cremoso produzido com massa obtida por acidificação direta a quente e por ultrafiltração, SILVA et al. [13] utilizaram a "escala do ideal" [9] para avaliação dos atributos cremosidade, sabor salgado relativo, sabor ácido, preferência relativa e sabor característico de requeijão. Também foi empregada escala hedônica de 7 pontos para avaliação da aceitação geral entre as amostras. Os resultados indicaram que o requeijão fabricado com retentado da ultrafiltração do leite foi semelhante, e em alguns atributos (sabor característico e cremosidade) superior ao tradicional na preferência do consumidor.

Apesar dos conhecimentos desenvolvidos, ainda não foi proposto um perfil dos atributos sensoriais que descrevam a qualidade do requeijão. Assim, este trabalho teve a finalidade de desenvolver esse perfil, através do método da Análise Descritiva Quantitativa, utilizando quatro marcas comerciais, bem como avaliar a aceitabilidade desses produtos.

\section{2 - MATERIAL E MÉTODOS}

\section{1 - Amostras}

Foram avaliadas quatro marcas comerciais de requeijão envasadas em copos de vidro de $250 \mathrm{~mL}$, adquiridas em supermercados da região de Campinas - SP. Os testes sensoriais foram realizados no Laboratório de Análise Sensorial da Faculdade de Engenharia de Alimentos (FEA/ UNICAMP), cujas instalações incluem cabinas individuais e controle de iluminação e temperatura ambiente.

O perfil sensorial de cada amostra de requeijão foi determinado utilizando a Análise Descritiva Quantitativa (ADQ) descrita por STONE \& SIDEL [14], com provadores treinados. Foi ainda realizado um teste de aceitação com a participação de consumidores do produto, conforme recomendações de MEILGAARD et al. [9].

\section{2 - Análise descritiva quantitativa}

\subsection{1 - Recrutamento e pré-seleção dos provadores}

Os provadores foram recrutados dentre os alunos e funcionários da FEA que já haviam sido selecionados em equipes sensoriais anteriores em função de seu desempenho em testes de reconhecimento de odor e gostos básicos [2].

\subsection{2 - Desenvolvimento da terminologia descri- tiva e treinamento dos provadores}

Para o levantamento inicial de termos que descrevessem a qualidade do requeijão, foram escolhidas 3 amostras que representavam o universo de variação entre as amostras a serem analisadas, ou seja, foram escolhidas amostras que apresentavam diferenças marcantes de consistência e sabor. Utilizando o método de Rede, segundo MOSKOWITZ [10], as 3 amostras foram apresentadas simultaneamente, solicitando-se ao provador que descrevesse as similaridades e as diferenças entre elas. Foi utilizada uma ficha para aparência, aroma, sabor e textura, e outra para manuseio (Figuras 1 e 2).

NOME
AMOSTRAS
Você está recebendo três amostras de requeijão. Inicialmente identifique
as duas amostras mais similares com relação a aparência e descreva, com
detalhes, em que elas são similares e como elas diferem da terceira amostra. Em
seguida repita o mesmo procedimento com relação ao aroma, sabor e textura.
Similaridades
APARÊNCIA
AROMA
SABOR
TEXTURA

FIGURA 1. Ficha utilizada para o Método Rede no levantamento dos termos descritivos.

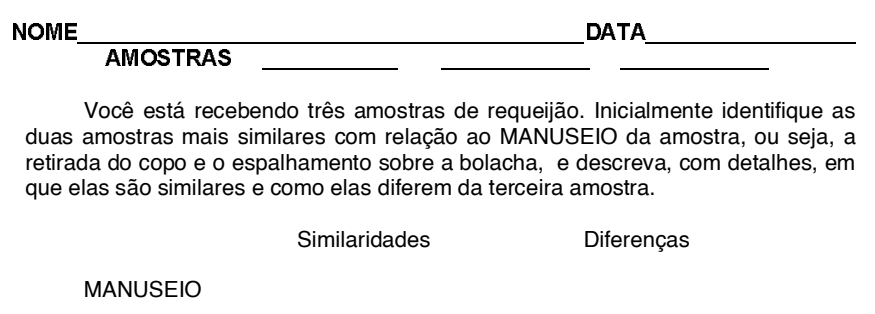

FIGURA 2. Ficha utilizada no Método Rede para levantar termos de manuseio.

Após cada provador ter gerado seus próprios termos, foi realizada uma discussão em grupo, sob a supervisão de um moderador, com o objetivo de agrupar termos semelhantes e eliminar aqueles que não eram percebidos pela maioria dos provadores. Em 03 sessões posteriores de avaliação das amostras e de referências foi elaborada uma Ficha de Avaliação contendo os termos descritivos escolhidos em consenso pela equipe sensorial. Nessa ficha (Figura 3) foi utilizada uma escala linear não estruturada de $9 \mathrm{~cm}$, ancorada nas suas extremidades com termos que expressam intensidade. Quando o descritor tinha a possibilidade de não estar presente em alguma das amostras, o extremo inferior da escala era chamado "nenhum". Nessa etapa foi também elaborada uma lista de definições dos termos descritivos, com as 
respectivas referências para melhor uniformizar a avaliação dos provadores (Figura 4).

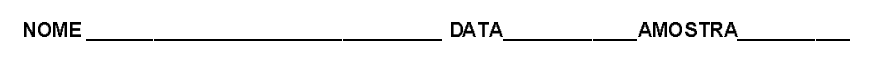

Por favor, prove a amostra e avalie a intensidade percebida para cada atributo colocando um traço vertical nas escalas correspondentes.

APARÊNCIA
Cor amarela
Liso
USO
Fonsistência
Esparmação de fio

FIGURA 3. Ficha utilizada no treinamento e avaliação das amostras de requeijão.

\subsection{3 - Seleção final da equipe de ADQ}

Nesta etapa foram avaliadas três amostras de requeijão, em delineamento experimental de blocos completos casualizados com três repetições. Os provadores utilizaram a própria Ficha de Avaliação desenvolvida na etapa anterior, sendo-lhes permitido consultar, a qualquer momento da sua análise, a lista de definições e as referências.

Foi então realizada a Análise de Variância para os resultados de cada provador, para cada descritor avaliado, tendo como fontes de variação amostras e repetições. Foram selecionados, para compor a equipe descritiva final, aqueles provadores que apresentaram bom poder discriminativo $\left(\mathrm{p}_{\text {amostra }}<0,50\right)$; consenso com os demais membros do grupo e boa reprodutibilidade nos julgamentos $\left(p_{\text {repeticão }}>0,05\right)$, segundo metodologia proposta por DAMASIO \& COSTELL [5].

\subsection{4 - Avaliação das amostras}

O experimento constou da avaliação sensorial de quatro amostras de requeijão, por uma equipe de nove provadores treinados. Foi utilizado o delineamento em blocos completos casualizados com 3 repetições, usando-se a técnica de apresentação monádica, ou seja, foi apresentada uma amostra de cada vez. A ordem de apresentação das amostras foi balanceada, de forma a evitar vícios nos resultados.

Os provadores receberam amostras de aproximadamente $20 \mathrm{~g}$ de requeijão a $20^{\circ} \mathrm{C}$, servidas em copinhos descartáveis codificados com números de três digitos,

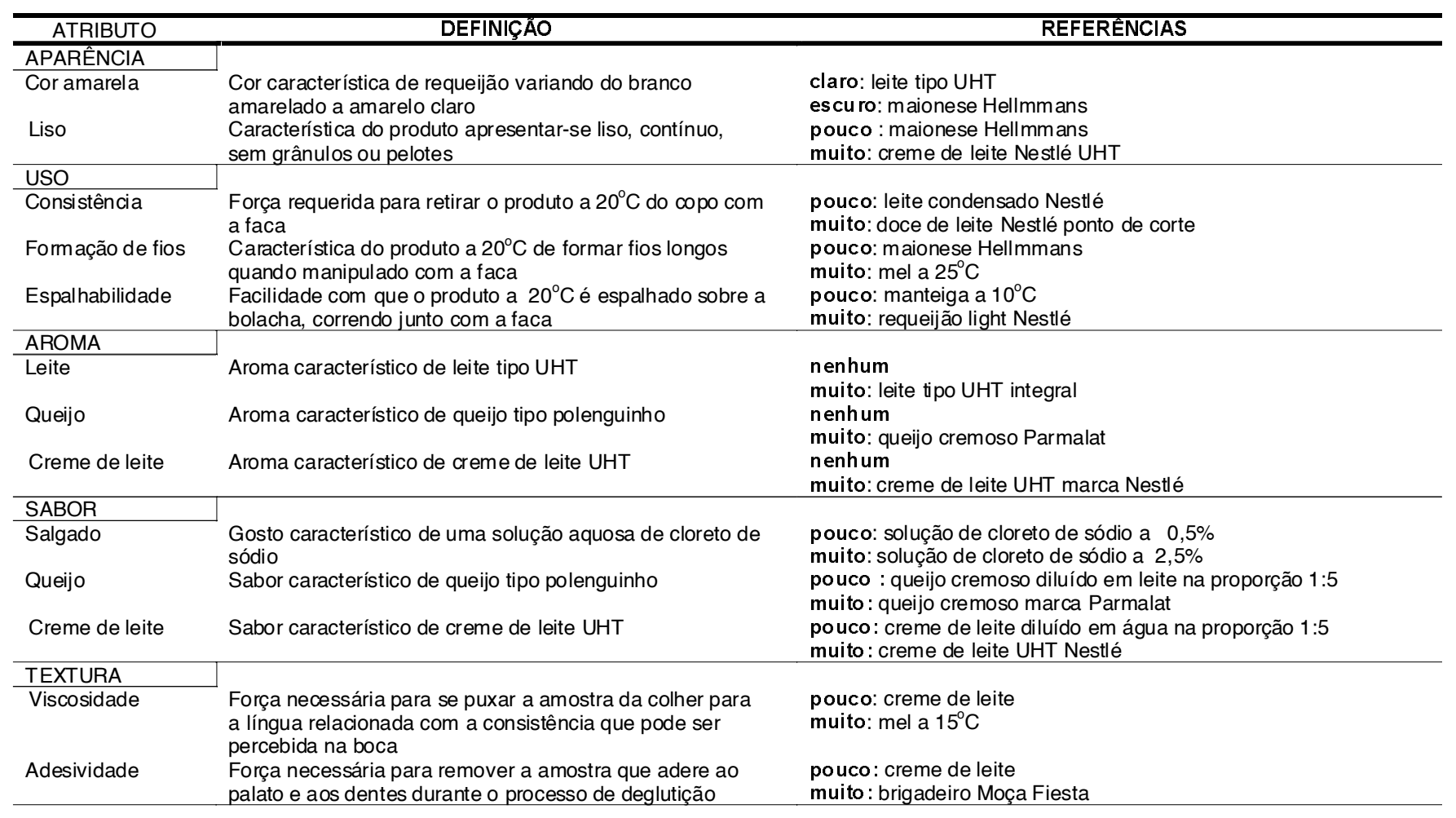

FIGURA 4. Lista de definições dos termos descritivos e respectivas referências. 
acompanhados de colherinhas para degustar o produto puro; um copo com água e uma bolacha "cream cracker" para eliminar resíduos de requeijão na boca. Foi fornecida também uma amostra do produto em copo tulipa tampado com vidro de relógio para facilitar a percepção do aroma.

Para avaliação de aparência e características de uso do produto, as amostras foram apresentadas na própria embalagem, todas ao mesmo tempo, sob luz do dia. Foi fornecido aos provadores uma faca para cada amostra, para possibilitar a manipulação do produto e o espalhamento sobre uma bolacha "cream cracker".

\subsection{5 - Teste de aceitação}

A aceitabilidade das quatro amostras de requeijão foi avaliada com a participação de 41 consumidores do produto recrutados verbalmente. $\mathrm{O}$ teste foi realizado em laboratório, sob condições controladas. Cada provador recebeu um copo de requeijão de cada vez, codificado com número de três dígitos aleatórios, sendo-lhe solicitado manusear o produto com uma faca, espalhar sobre uma fatia de torrada (tipo canapé), avaliar a amostra de uma forma global (impressão geral) e, em seguida, quanto ao manuseio e sabor, utilizando para isso uma escala hedônica estruturada mista de 9 pontos.

\subsection{6 - Análises estatísticas}

Os resultados da ADQ foram submetidos a Análise de Variância (ANOVA), Teste de Tukey ao nivel de 5\% de significância para a comparação entre as médias, e Análise de Componentes Principais. Os resultados do teste de aceitação foram analisados por meio de Análise de
Variância e teste de Tukey para médias. Todas as análises estatísticas foram realizadas utilizando-se o programa estatístico SAS [12].

\section{3 - RESULTADOS E DISCUSSÃO}

\section{1 - Seleção de provadores e termos descritivos}

O desempenho dos provadores pode ser analisado através dos valores de $\mathrm{p}_{\text {amostra }}$ e $\mathrm{p}_{\text {repetiçăo }}$ que são apresentados na Tabela 1. Observa-se que, de um modo geral, o desempenho dos provadores foi satisfatorio, pois aqueles que apresentaram menor capacidade discriminatória conseguiram diferenciar pelo menos 9 entre os 13 atributos analisados. Além disso foi verificado um bom consenso da equipe, sendo que alguns provadores apresentaram problemas isolados em atributos distintos, como foi o caso do provador 3 com aroma de leite e cor, o provador 5 com aroma de queijo e o provador $9 \mathrm{com}$ sabor salgado. Os provadores tiveram dificuldade em discriminar as amostras quanto a aroma de leite e aroma de queijo, indicando que, provavelmente, essas amostras realmente não diferiram quanto a esses atributos.

O provador 10, apesar de ter ótimo desempenho discriminatório, apresentou sério problema de repetibilidade e falta de consenso para quatro descritores, sendo portanto eliminado. O descritor espalhabilidade provocou, mesmo após treinamento, uma certa confusão entre os provadores, que não conseguiram chegar a um consenso em suas análises, dividindo-se em dois grupos distintos quanto à ordem das amostras em intensidade desse atributo. Decidiu-se, então eliminar esse descritor.

TABELA 1. Desempenho dos provadores candidatos à equipe sensorial em relação a seu poder discriminatório ( $\left.p_{\text {amostra }}\right)$ e repetibilidade $\left(\mathrm{p}_{\text {repetição }}\right)$. Valores desejáveis: $\mathrm{p}_{\text {amostra }}<0,50$ e $\mathrm{p}_{\text {repetiçăo }}>0,05$.

\begin{tabular}{|c|c|c|c|c|c|c|c|c|c|c|c|c|c|}
\hline \multicolumn{2}{|c|}{ DESCRITOR } & \multirow[t]{2}{*}{$\mathbf{P}$} & \multicolumn{11}{|c|}{ PROVADOR } \\
\hline & & & 1 & 2 & 3 & 4 & 5 & 6 & 7 & 8 & 9 & 10 & 11 \\
\hline \multirow{8}{*}{ APARÊNCIA } & Cor & $\mathrm{pam}_{\mathrm{am}}$ & 0,176 & $0,563^{\star}$ & 0,088 & 0,254 & 0,023 & 0,066 & 0,010 & 0,253 & 0,038 & 0,011 & 0,177 \\
\hline & & prep & 0,355 & 0,477 & 0,165 & 0,293 & $0,038^{\star}$ & 0,144 & $0,022^{*}$ & 0,271 & $0,038^{\star}$ & $0,031^{*}$ & 0,382 \\
\hline & Liso & $p_{a m}$ & 0,021 & 0,001 & 0,001 & 0,125 & 0,002 & $0,992^{*}$ & 0,123 & 0,008 & 0,013 & 0,031 & $0,547^{*}$ \\
\hline & Consistência & Pam & $0,922^{*}$ & 0,130 & 0,176 & $0,689^{\star}$ & $0,868^{\star}$ & 0,268 & $0,737^{\star}$ & 0,023 & 0,052 & $0,513^{\star}$ & 0,025 \\
\hline & & prep & 0,564 & 0,264 & 0,120 & 0,531 & 0,276 & 0,373 & 0,799 & 0,061 & 0,090 & 0,777 & $0,047^{\star}$ \\
\hline & Formação fio & $\mathrm{pam}_{\mathrm{m}}$ & 0,001 & 0,001 & 0,060 & 0,001 & 0,001 & 0,026 & 0,001 & 0,001 & 0,001 & 0,017 & 0,001 \\
\hline & & prep & $0,004^{*}$ & $0,001^{*}$ & 0,109 & $0,001^{*}$ & $0,001^{*}$ & 0,070 & $0,001^{*}$ & $0,001^{*}$ & $0,005^{*}$ & $0,043^{*}$ & $0,001^{*}$ \\
\hline & & prep & 0,404 & 0,696 & 0,238 & 0,257 & $0,002^{*}$ & 0,143 & 0,076 & 0,121 & 0,379 & $0,032^{*}$ & $0,001^{*}$ \\
\hline \multirow[t]{6}{*}{ AROMA } & Leite & $\mathrm{Pam}_{\mathrm{a}}$ & $0,559^{\star}$ & 0,444 & 0,042 & $0,547^{\star}$ & 0,000 & $0,654^{*}$ & $0,919^{\star}$ & $0,937^{\star}$ & $0,513^{\star}$ & 0,015 & 0,444 \\
\hline & & Prep & 0,576 & 0,500 & 0,075 & 0,733 & 0,000 & 0,626 & 0,898 & 0,917 & 0,737 & $0,042^{\star}$ & 0,500 \\
\hline & Queijo & pam & $0,712^{*}$ & $0,953^{*}$ & $0,652^{*}$ & $0,768^{*}$ & $0,803^{*}$ & 0,385 & $0,518^{*}$ & 0,063 & 0,336 & 0,012 & $0,917^{\star}$ \\
\hline & & prep & 0,552 & 0,937 & 0,684 & 0,979 & 0,561 & 0,473 & 0,776 & 0,143 & 0,390 & $0,030^{*}$ & 0,823 \\
\hline & Creme leite & pam & 0,303 & 0,137 & 0,431 & $0,985^{\star}$ & 0,380 & 0,414 & 0,378 & 0,128 & 0,381 & 0,085 & $0,391^{\star}$ \\
\hline & & prep & $0,010^{\star}$ & 0,176 & 0,330 & 0,349 & $0,022^{*}$ & 0,428 & 0,588 & 0,263 & 0,494 & 0,052 & 0,312 \\
\hline \multirow{5}{*}{ SABOR } & Salgado & pam & 0,127 & 0,062 & 0,251 & 0,028 & 0,001 & 0,036 & 0,116 & 0,181 & 0,033 & 0,001 & 0,492 \\
\hline & & prep & 0,095 & 0,108 & 0,495 & 0,059 & $0,001^{\star}$ & $0,039^{\star}$ & 0,261 & 0,250 & 0,082 & $0,003^{\star}$ & 0,766 \\
\hline & Queijo & $p_{a m}$ & 0,363 & $0,898^{\star}$ & 0,056 & 0,032 & 0,120 & $0,740^{*}$ & 0,019 & $0,564^{*}$ & 0,092 & 0,006 & 0,045 \\
\hline & Creme & pam & 0,260 & 0,011 & 0,225 & 0,292 & 0,431 & 0,324 & 0,017 & 0,178 & 0,164 & 0,105 & 0,00 \\
\hline & de leite & prep & 0,418 & $0,028^{*}$ & 0,303 & 0,432 & 0,540 & 0,317 & $0,031^{*}$ & 0,101 & 0,111 & 0,151 & 0,00 \\
\hline \multirow[t]{6}{*}{ TEXTURA } & Viscosidade & $\mathrm{Pam}_{\mathrm{am}}$ & $0,988^{*}$ & 0,253 & $0,791^{\star}$ & 0,239 & 0,245 & $0,550^{\star}$ & 0,292 & $0,619^{\star}$ & 0,122 & 0,003 & 0,175 \\
\hline & & prep & 0,637 & 0,329 & 0,702 & 0,390 & 0,186 & 0,556 & 0,500 & 0,714 & $0,025^{*}$ & $0,008^{*}$ & 0,326 \\
\hline & Adesividade & pam & $0,651^{*}$ & 0,402 & $0,918^{*}$ & 0,352 & $0,704^{*}$ & 0,140 & 0,056 & $0,704^{\star}$ & $0,714^{*}$ & 0,174 & 0,369 \\
\hline & & $p_{\text {rep }}$ & 0,763 & 0,547 & 0,588 & 0,417 & 0,564 & 0,056 & 0,119 & 0,892 & 0,161 & 0,286 & 0,659 \\
\hline & ND & & 5 & 3 & 3 & 4 & 3 & 4 & 3 & 4 & 3 & 1 & 2 \\
\hline & NR & & 2 & 3 & 1 & 1 & 6 & 1 & 4 & 2 & 4 & 8 & 3 \\
\hline
\end{tabular}

$*$ = valores não atendendo aos valores especificados para $\mathrm{p}_{\text {amostra }}$ e $\mathrm{p}_{\text {repetiçäa }}, 0,50$

$\mathrm{ND}=$ número de vezes que o provador não discriminou as amostras a $\mathrm{p}<, 0,50$
$\mathrm{NR}=$ número de vezes que o provador não apresentou repetibilidade a $\mathrm{p}>0,05$ 
Por sua vez, o descritor formação de fios apresentou problema de repetibilidade para 9 dentre os 11 provadores avaliados, necessitando de nova sessão de treinamento.

\section{2 - Perfil sensorial do requeijão}

Os resultados da Análise de Variância das notas atribuidas pelos provadores a cada descritor, para cada uma das marcas de requeijão avaliadas sensorialmente, são apresentados na Tabela 2 . O perfil de cada uma dessas marcas é mostrado graficamente na Figura 5, onde o valor médio atribuído pelos provadores a cada descritor é marcado no eixo correspondente. O centro da figura representa o ponto zero da escala utilizada na avaliação, enquanto a intensidade aumenta do centro para a periferia. Assim, o perfil sensorial se revela quando se faz a conexão dos pontos.

Observou-se que o requeijão da marca A distinguiu-se dos demais por apresentar aparência muito lisa, alta formação de fio e aroma de leite mais intenso. A amostra B destacou-se por seu sabor salgado e sabor de queijo. A marca C, por sua vez, caracterizou-se por apresentar quase nenhuma formação de fio, além de apresentar maior consistência, adesividade, viscosidade, cor e sabor de creme de leite. O requeijão da marca $\mathrm{D}$ apresentou comportamento muito semelhante à marca $\mathrm{C}$, diferindo mais em relação à cor amarela menos intensa e maior intensidade de sabor salgado.

TABELA 2. Médias dos valores atribuídos pelos provadores a cada descritor, para as amostras de requeijão.

\begin{tabular}{|c|c|c|c|c|}
\hline \multirow[t]{2}{*}{ Descritores } & \multicolumn{4}{|c|}{ Amostras } \\
\hline & A & B & C & D \\
\hline Cor amarela & $2,68^{c}$ & $3,99^{\mathrm{ab}}$ & $4,82^{\mathrm{a}}$ & $3,45^{\mathrm{bc}}$ \\
\hline Liso & $7,73^{a}$ & $4,25^{b}$ & $2,68^{\mathrm{c}}$ & $3,14^{c}$ \\
\hline Consistência & $2,56^{c}$ & $4,00^{\mathrm{b}}$ & $5,82^{a}$ & $4,94^{\mathrm{ab}}$ \\
\hline Formação de fio & $7,86^{\mathrm{a}}$ & $2,92^{\mathrm{b}}$ & $0,74^{\mathrm{c}}$ & $0,97^{\circ}$ \\
\hline Aroma de leite & $3,96^{\mathrm{a}}$ & $2,73^{b}$ & $2,75^{\mathrm{ab}}$ & $2,92^{\mathrm{ab}}$ \\
\hline Aroma creme de leite & $2,17^{\mathrm{a}}$ & $2,15^{\mathrm{a}}$ & $2,85^{\mathrm{a}}$ & $3,18^{\mathrm{a}}$ \\
\hline Salgado & $5,22^{b}$ & $7,07^{\mathrm{a}}$ & $2,58^{d}$ & $4,20^{\mathrm{c}}$ \\
\hline Sabor queijo & $4,20^{\mathrm{a}}$ & $4,73^{\mathrm{a}}$ & $4,20^{\mathrm{a}}$ & $4,57^{\mathrm{a}}$ \\
\hline Sabor creme de leite & $2,59^{\mathrm{a}}$ & $2,75^{a}$ & $3,33^{\mathrm{a}}$ & $2,78^{a}$ \\
\hline Viscosidade & $3,90^{\mathrm{bc}}$ & $3,31^{c}$ & $4,99^{\mathrm{a}}$ & $4,4^{\mathrm{ab}}$ \\
\hline Adesividade & $3,08^{b}$ & $3.17^{\mathrm{b}}$ & $5,15^{\mathrm{a}}$ & $4,71^{\mathrm{a}}$ \\
\hline
\end{tabular}

Médias na mesma linha acompanhadas de mesma letra não diferem entre si ao nível de $5 \%$ de significância

Quando os resultados da Análise Descritiva Quantitativa do requeijão foram submetidos à Análise Multivariada de Componentes Principais (ACP), as características sensoriais de cada amostra foram evidenciadas, apresentando grande concordância com os resultados obtidos anteriormente. No gráfico da ACP (Figura 6), os descritores são representados como vetores, os quais caracterizam as amostras que se localizam próximas a eles. As amostras são representadas por triângulos, sendo cada vértice uma repetição. Assim, a amostra de marca A apresentou maior intensidade de formação de fio e aparência lisa, enquanto que a amostra B caracterizou-se por maior intensidade de sabor queijo e sabor salgado. A amostra C foi caracterizada por apresentar elevada adesividade e consistência, enquanto que a amostra da marca D apresentou uma contribuição mais equilibrada desses descritores. Esta última amostra também caracterizou-se pelo sabor e aroma de creme de leite, maior viscosidade e cor mais intensa.

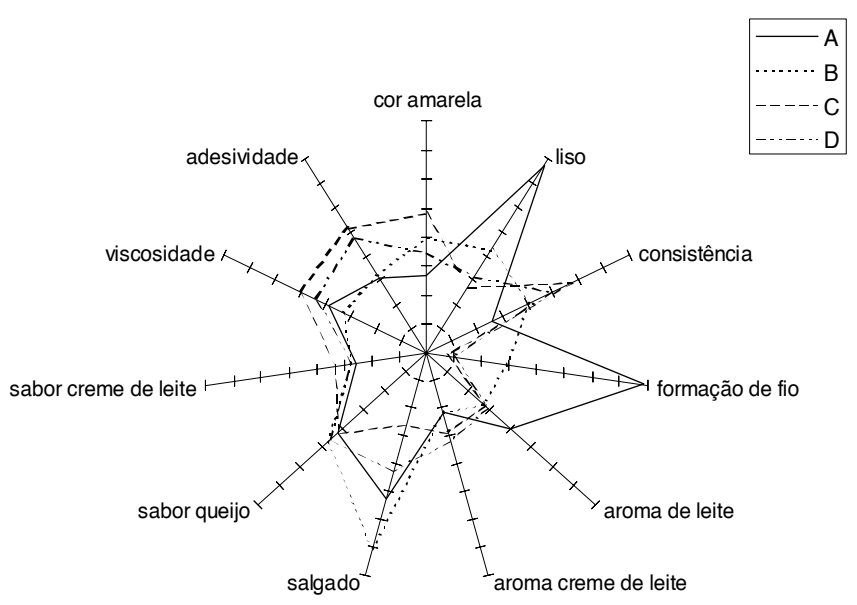

FIGURA 5. Perfil sensorial das amostras de requeijão cremoso.

Nesse tipo de gráfico, quanto maior a decomposição do vetor nos eixos dos componentes, maior sua importância para caracterizar as diferenças entre as amostras. Observou-se, nesse caso, que para explicar os $54 \%$ da variabilidade entre as amostras devidos ao primeiro Componente Principal (CP1), foi importante a contribuição da maioria dos descritores analisados. A pouca variabilidade explicada pelo segundo CP foi devido principalmente ao sabor de queijo, aroma de leite, sabor de creme de leite e salgado.

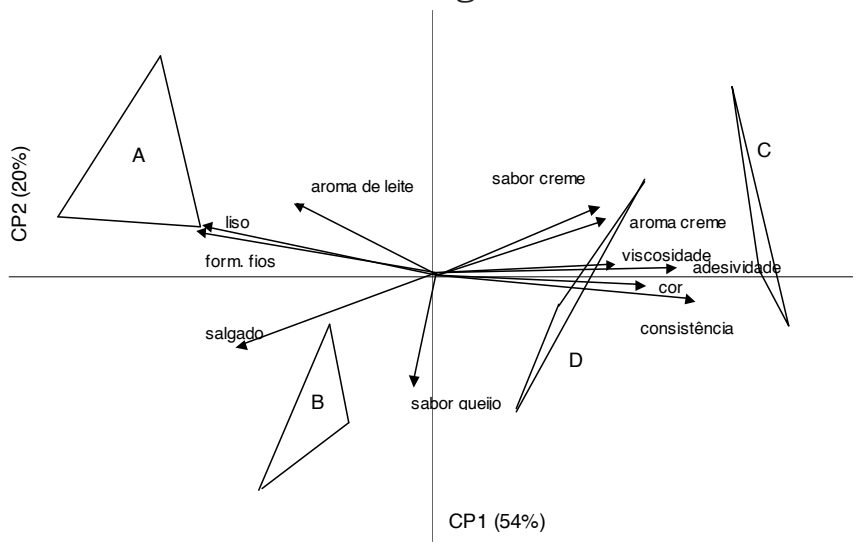

FIGURA 6. Projeção dos descritores sensoriais e amostras de Requeijão cremoso nas duas primeiras Componentes Principais.

De fato, os testes de ANOVA e Tukey (Tabela 2) mostraram que não houve diferença significativa $(p<0,05)$ entre as amostras, somente para os atributos aroma creme de leite, sabor creme de leite e sabor queijo. O atri- 
buto aroma de queijo foi excluído, pois apresentou interação amostra x provador significativa e provocou falta de consenso entre os provadores.

Na Tabela 3 são apresentados os coeficientes de correlação de Pearson entre os 11 descritores de requeijão para as quatro marcas estudadas. Observouse alta correlação positiva significativa $(p<0,01)$ entre $o$ descritor consistência com os descritores cor e adesividade; entre formação de fios e liso; entre viscosidade e adesividade, vetores que estão muito próximos entre si na configuração bidimensional da ACP (Figura 6). Por outro lado, os descritores consistência e adesividade apresentaram alta correlação negativa com formação de fios e liso, sugerindo que o aumento da consistência do requeijão por meio de espessantes pode estar inibindo a característica da massa de queijo fundida de formar fios, e provocando a formação de grumos que aderem ao palato e aos dentes. Através dos resultados das correlações entre os vetores, observou-se também que as amostras com maior adesividade e viscosidade apresentaram-se menos salgadas (alta correlação negativa com o descritor salgado).

\section{3 - Teste de aceitabilidade}

Através da análise dos resultados do teste de aceitação (Tabela 4) observou-se que as amostras de

TABELA 3. Matriz de correlação entre os descritores de requeijão para as quatro marcas avaliadas.

\begin{tabular}{|c|c|c|c|c|c|c|c|c|c|c|c|}
\hline Vetor & Consistência & $\begin{array}{l}\text { Formação } \\
\text { de fios }\end{array}$ & Aroma leite & $\begin{array}{l}\text { Aroma creme } \\
\text { de leite }\end{array}$ & Salgado & $\begin{array}{l}\text { Sabor } \\
\text { queijo }\end{array}$ & $\begin{array}{l}\text { Sabor } \\
\text { creme de } \\
\text { leite }\end{array}$ & Cor & Liso & Viscosidade & Adesividade \\
\hline Consistência & 1,000 & $-0,938^{* *}$ & $-0,541^{\mathrm{ns}}$ & $0,497^{\text {ns }}$ & $-0,595^{\star}$ & $0,042^{\text {ns }}$ & $0,461^{\mathrm{ns}}$ & $0,779^{\star *}$ & $-0,947^{\star *}$ & $0,643^{*}$ & $0,813^{\star *}$ \\
\hline Formação de fios & & 1,000 & $0,628^{*}$ & $-0,548^{\text {ns }}$ & $0,396^{\mathrm{ns}}$ & $-0,215^{\mathrm{ns}}$ & $-0,397^{\mathrm{ns}}$ & $-0,711^{\star *}$ & $0,990^{\star *}$ & $-0,447^{\mathrm{ns}}$ & $-0,729^{\star *}$ \\
\hline Aroma leite & & & 1,000 & $-0,042^{\text {ns }}$ & $0,112^{\mathrm{ns}}$ & $-0,181^{\mathrm{ns}}$ & $-0,022^{n s}$ & $-0,541^{\mathrm{ns}}$ & $0,591^{*}$ & $-0,145^{\mathrm{ns}}$ & $-0,455^{\mathrm{ns}}$ \\
\hline $\begin{array}{l}\text { Aroma creme de } \\
\text { leite }\end{array}$ & & & & 1,000 & $-0,619^{*}$ & $-0,346^{\mathrm{ns}}$ & $0,627^{*}$ & $0,292^{\text {ns }}$ & $-0,496^{\mathrm{ns}}$ & $0,261^{\mathrm{ns}}$ & $0,575^{\mathrm{ns}}$ \\
\hline Salgado & & & & & 1,000 & $0,491^{\mathrm{ns}}$ & $-0,579^{\star}$ & $-0,426^{\mathrm{ns}}$ & $0,371^{\mathrm{ns}}$ & $-0,779^{\star \star}$ & $-0,824^{\star *}$ \\
\hline Sabor queijo & & & & & & 1,000 & $-0,455^{\mathrm{ns}}$ & $-0,163^{\mathrm{ns}}$ & $-0,247^{\mathrm{ns}}$ & $0,022^{\text {ns }}$ & $-0,018^{\mathrm{ns}}$ \\
\hline $\begin{array}{l}\text { Sabor creme de } \\
\text { leite }\end{array}$ & & & & & & & 1,000 & $0,627^{\star}$ & $-0,365^{\mathrm{ns}}$ & $0,202^{\mathrm{ns}}$ & $0,438^{\text {ns }}$ \\
\hline Cor & & & & & & & & 1,000 & $-0,723^{\star *}$ & $0,364^{\mathrm{ns}}$ & $0,512^{\mathrm{ns}}$ \\
\hline Liso & & & & & & & & & 1,000 & $-0,460^{\mathrm{ns}}$ & $-0,714^{\star *}$ \\
\hline Viscosidade & & & & & & & & & & 1,000 & $0,788^{\star *}$ \\
\hline Adesividade & & & & & & & & & & & 1,000 \\
\hline
\end{tabular}

ns = não significativo ao nivel de $\mathrm{p}<0,05$ de probabilidade

* significativo ao nivel $\mathrm{p}<0,05$ de probabilidade

** significativo ao nivel $p<0,01$ de probabilidade

requeijão foram igualmente aceitas pelos provadores quanto a sabor e impressão geral. Houve diferença significativa a $\mathrm{p}<0,05$ somente para o manuseio, sendo que as marcas $\mathrm{B}$ e $\mathrm{C}$ foram mais aceitas que a amostra $A$, não havendo diferença entre as amostras B e D. Consultando a Tabela 2, foi observado que os provadores gostaram mais das amostras mais consistentes e com menos formação de fio, indicando que o consumidor aprecia menos o requeijão mole, que pinga da faca.

TABELA 4. Médias dos valores atribuídos pelos provadores as quatro marcas de requeijão, em uma escala hedônica de 9 pontos.

\begin{tabular}{lcccc}
\hline Atributo & Marca A & Marca B & Marca C & Marca D \\
\hline \hline Impressão geral & $7,07^{\mathrm{a}}$ & $6,83^{\mathrm{a}}$ & $7,51^{\mathrm{a}}$ & $6,98^{\mathrm{a}}$ \\
Manuseio & $5,46^{\mathrm{c}}$ & $7,44^{\mathrm{ab}}$ & $7,93^{\mathrm{a}}$ & $7,10^{\mathrm{b}}$ \\
Sabor & $7,07^{\mathrm{a}}$ & $7,24^{\mathrm{a}}$ & $6,63^{\mathrm{a}}$ & $6,95^{\mathrm{a}}$ \\
\hline
\end{tabular}

Médias na mesma linha acompanhadas de mesma letra não diferem entre si ao nivel de $5 \%$ de significância

\section{4 - CONCLUSÕES}

- Um perfil sensorial para o requeijão cremoso, consistindo em descritores de aparência, aroma, sabor e textura, foi estabelecido utilizando-se um conjunto de amostras comerciais. Dos treze descritores analisados, cor, consistência (aparência), formação de fio, aroma de leite, salgado, viscosidade e adesividade foram considerados importantes na determinação da qualidade do produto, pois discriminaram claramente amostras de marcas distintas.

- O requeijão da marca A caracterizou-se por apresentar baixa consistência e alta formação de fio, além de uma aparência lisa, sem grumos (homogênea), enquanto que a caracteristica predominante da marca B foi o sabor salgado muito intenso. Os produtos das marcas $\mathrm{C}$ e D apresentaram perfis sensoriais semelhantes e opostos à marca A, sendo mais consistentes, pouco lisos e com pouca formação de fio. 
- Os atributos de aroma e de sabor de leite, creme de leite e queijo não variaram significativamente entre as amostras avaliadas e portanto não foram considerados importantes para determinar a qualidade sensorial de requeijão.

- Todas as marcas de requeijão analisadas foram igualmente muito bem aceitas pelos consumidores, em relação a sua qualidade geral e sabor. No entanto a aceitação dos consumidores foi baseada no manuseio do produto, os quais gostaram mais das amostras mais consistentes e com menor formação de fio.

\section{5 - REFERÊNCIAS BIBLIOGRÁFICAS}

[1] ANZALDUA-MORALES, A. La evaluación sensorial de los alimentos en la teoria y en la practica. Zaragoza: Acribia. 1994. 198p.

[2] ASTM-AMERICAN SOCIETY FOR TESTING AND MATERIALS Guidelines for the Selection and Training of Sensory Panel Members. Philadelphia: ASTM, 1981. 77p. (STP 758)

[3] BRASIL. Ministério da Agricultura. Regulamentos técnicos de identidade e qualidade de leite e produtos lácteos. Brasília, 1997. 77p.

[4] CASTRO, A.F.; PRESTES, M.M.; RIBEIRO, L.; IMIANOVSKY, U.; REITER, M.G.R. Qualidade sensorial e microbiológica do requeijão tradicional e light. Revista do Instituto de Laticínios Cândido Tostes, v. 57, n. 327, p. 160162, 2002.

[5] DAMASIO, M.H.; COSTELL, E. Análisis sensorial descriptivo: generación de descriptores y selección de catadores. Revista Agroquimica de Technologia de Alimentos, v. 31,n. 2, p. 165-78, 1991.

[6] FERNANDES, A.G.; DO VALE, J.L.E.; CAMPOS, S.D.S.; MORI, E.E.M. Formulação de sais emulsificantes para a elaboração de requijão cremoso e outros queijos fundidos. Revista do Instituto de Laticínios Cândido Tostes, v. 40, n. 237, p. 27-43, 1986.
[7] FOODBASE. Base de dados em CD-ROM da Legislação Brasileira. Associação Brasileira das Indústrias de Alimentos, 1996.

[8] GIGANTE, M.L.; ALMEIDA, T.C.A.; KAWASHIMA, L.M.; ROIG, S.M. Sensory evaluation of "Requeijão cremoso" cheese with protein concentrate. In: 1999 IFT ANNUAL MEETING. Chicago, E.U.A., 1999. Summarys. http:// www.confex.com/ift/99annual/abstracts/3753.htm. 08 de dezembro de 2002.

[9] MEILGAARD, M.; CIVIlle, G.V.; CARR, B.T. Sensory evaluation tecniques. Boca Raton: CRC Press, 1987. $2 \mathrm{v}$.

[10] MOSKOWITZ, H.R. Product testing and sensory evaluation of foods: marketing and $R \& D$ approaches. Westport: Food and Nutrition Press, 1983. 605p.

[11] MUNCK, A.V.; LOURENÇO NETO, J.P. M.; FURTADO, M.M. Fabricação de requeijão cremoso a partir de ácido cítrico e citrato de sódio. Leite e Derivados, v. 2, n. 9, p. 4749, 1992.

[12] SAS User's procedures guide. Version 6, Cary:SAS Institute, Inc 1989. $2 \mathrm{v}$.

[13] SILVA, A.T.; VAN DENDER, A.G.F.; MORI, E.M.; MORENO, I.; YOTSUYANAGI, K. Avaliação comparativa das características do requeijão cremoso produzido com massa obtida por acidificação direta a quente e por ultrafiltração. Revista do Instituto Cândido Tostes, v. 57, n. 327, p. 263-265, 2002.

[14] STONE, H.; SIDEL, J.L. Descriptive analysis. In: STONE, H.; SIDEL, J.L. Sensory Evaluation Practices. London: Academic Press. 1985, p. 202-226.

[15] VAN DENDER, A.G.F. Princípios básicos da fabricação de queijos fundidos de requeijão cremoso. Leite e Derivados,v. 1, n. 3, p. 26, 1992.

[16] VAN DENDER, A.G.F. Evaluation of the stability of "Requeijão cremoso" in different packages. In: 2001 IFT Annual Meeting, New Orleans, E.U.A., 2001. http:// ift.confex.com/ift/2001/techprogram/paper_7943.htm. $08 / 12 / 2002$ 